\title{
Heterogene Stadträume der Globalisierung
}

\author{
Slums in Texten Meineckes, Tawadas und Trojanows
}

\section{Christoph Schaub}

\begin{abstract}
Against the backdrop of a new transnational discourse about slums since the early 2000s, the article analyses representations of slums located in South Africa, India, and Brazil in texts of three contemporary writers: Thomas Meinecke, Yoko Tawada, and Ilija Trojanow. Their works are engaged in a positive recoding of the cultures of slums as sites of multilingualism, hybridity, and cosmopolitanism. They provide alternative literary representations of slums and add to the existing knowledge about them. The article identifies narrative, thematic, and cultural-political elements that are characteristic for these authors' representations of slums. These elements may serve as steppingstones for a more comprehensive treatment of such heterogenous urban spaces of globalization in literary studies.
\end{abstract}

Title: Heterogenous Urban Spaces of Globalization: Slums in Meinecke, Tawada, and Trojanow

Keywords: globalization; slum; urban culture; contemporary German literature; travel literature

Seit der Neupopularisierung des Begriffs >Slum < durch die von der UNO und der Weltbank geförderte Initiative Cities without Slums (2000) und Mike Davis' Planet of Slums (2007) kann diese urbane Formation als einer der paradigmatischen Räume der Globalisierung des späten 20. und frühen 21. Jahrhunderts gelten, zu denen außerdem die Megastädte des Globalen Südens und die in Europa, Nordamerika und Ostasien gelegenen global cities zu zählen sind (vgl. Sassen 1991; World Bank/UNCHS 2000; Davis 2007; Otto 2015). Als Resultate von Globalisierungsprozessen sind Slums auch von Autor※innen der deutschsprachigen Gegenwartsliteratur registriert worden. Die hier diskutierten Texte von Thomas Meinecke, Yoko Tawada und Ilija Trojanow entwerfen die Slums Brasiliens, Südafrikas und Indiens nämlich als Knotenpunkte transnationaler und transkultureller Prozesse. Dabei werden Slums als positiv besetzte, heterogene Orte der Hybridität, Multilingualität und kosmopolitischen Alltagspraxis konstruiert. Meinecke, Tawada und Trojanow erzeugen auf diese Weise ein kulturtheoretisch erweitertes literarisches Wissen über Slums. ${ }^{1}$ Indem mein Aufsatz

1 Ich verstehe literarisches Wissen hier im Anschluss an Ottmar Ette (vgl. 2016), der dieses besonders durch die grenzüberschreitende Mobilität von Literatur entstehen sieht. Durch diese Mobilität kommen die Wissensbestände verschiedener Gesell- 
Konstruktionen von Slums in Texten dieser Autor*innen analysiert, trägt er zur Rekonstruktion eines literarischen Wissens von Slums in der deutschsprachigen Gegenwartsliteratur bei.

Die Bezugsgröße Slum verstehe ich hier in einem doppelten Sinne. Zum einen dient sie als übergreifender Sammelbegriff für eine große Zahl von national, regional und lokal unterschiedlich ausgeprägten und zudem jeweils in sich heterogenen großstädtischen Vierteln der Armen und Prekären, welche durch schlechte Lebensbedingungen und eine von sozialer und kultureller Marginalisierung geprägte Lebenswirklichkeit gekennzeichnet sind. Zum anderen verstehe ich Slum als eine diskursive Kategorie, die diese Räume der Armen und Prekären auf eine bestimmte, in der Regel stereotypisierende und pathologisierende Weise kulturell erfindet und dabei homogenisiert. Beispiele für Slums reichen vom Londoner Viertel Whitechapel im 19. Jahrhundert über proletarische Bezirke Berlins in den $1920 e r$ Jahren zu Chicagos South Side, Kairos Stadt der Toten oder Mumbais Dharavi in der Gegenwart. Seit der zweiten Hälfte des 20. Jahrhunderts sind Slums, wie Davis in Planet of Slums gezeigt hat, allerdings fast ausschließlich ein Phänomen des Globalen Südens, wo beinahe 80 Prozent aller Städtebewohner*innen in Slums leben und ihre Zahl bis 2040 voraussichtlich auf zwei Milliarden gestiegen sein wird (vgl. Davis 2007: 23 u. 151). Zugleich verdeutlicht Davis, indem er sozialwissenschaftliches, ökologisches, städteplanerisches und ökonomisches Wissen synthetisiert, dass sich hinter dem Begriff eine Vielfalt von oft informellen Behausungen und Lebensbedingungen verbirgt. Dies betrifft u.a. die Architektur, die geographische Lage von Slums in Städten oder den Zugang zu Infrastruktur und materiellen Ressourcen (vgl. ebd.: 30). Funktioniert das Wort Slum bei Davis einerseits als ein analytischer Begriff, mit dem er eine transnationale urbane Ordnung beschreibt, verwendet er Slum andererseits als einen politischen Kampfbegriff. ${ }^{2}$ Die skandalisierende Kraft des Wortes nutzt er, um soziale Ungleichheit sowie das postkoloniale Fortdauern von rassistischen und imperialistischen Herrschaftsstrukturen $\mathrm{zu}$ benennen (vgl. ebd.: 7 u. 96). In seiner Streitschrift fehlt jedoch eine Auseinandersetzung mit den vermeintlichen Kulturen der Slums.

Diese mangelnde Aufmerksamkeit gegenüber der kulturellen Dimension des Diskurses über Slums ist problematisch, weil die Imaginations- und Diskursgeschichte der Slums seit dem 19. Jahrhundert immer auch Vorstellungen einer bestimmten Kultur hervorbringt. Diese Vorstellungen wurden nicht zuletzt durch eine literarische Tradition gespeist, die aus der Auseinandersetzung mit den Armen der entstehenden Industriemetropolen hervorging. Durch diese wurden proletarische Viertel mit Wissenskategorien, Bildern und ästhetischen Repräsentationsweisen erfasst und als homogene Räume konstruiert, die mit negativen Merkmalen verbunden waren: Slums galten zum Beispiel als Orte von

schaften in Kontakt, und es entsteht ein von "discursive or disciplinary specializations" (ebd.: xxi) relativ uneingeschränktes Wissen.

2 | Pike hat diesbezüglich von einer "symbolic power" (2016: 199) des Begriffs ,Slum gesprochen. 
Krankheit, Chaos und Zivilisationsferne. Die Erzeugung der Proletarier*innen Europas als homogene Gruppe war dabei untrennbar von einem kolonialen Kontext, insofern die Konstruktionen des Anderen im Eigenen parallel zum othering der Kolonisierten verliefen und sich oft der gleichen Bilder bedienten: Slums waren beispielsweise urbane Dschungel (vgl. Brantlinger 1985; Poore 2000; Lindner 2004; Kleeberg 2011; Pike 2016). Wie Alan Gilbert ohne Bezug auf diese literarische Tradition zeigt, schreiben sich diese problematischen Kodierungen in der Neupopularisierung des Begriffs fort. Die Konstruktion von Slums wird von Bildern der Gefahr, Kriminalität und Immoralität dominiert, während zugleich eine homogene Bevölkerung evoziert wird: »Too many observers [...] apply the term slum with broad strokes; it embraces any place that is problematic and any group of people that lives there is automatically included. [...] All slums are bad and everyone living in them must suffer from the debilitating subculture that slum life produces.« (Gilbert 2007: 704) Gilbert fordert deshalb ein neues Verständnis von Slums als heterogenen Orten, das die Innenperspektive der Bewohner*innen ernst nimmt: »As [Peter] Marris [...] points out: >A slum is only a slum in the eyes of someone for whom it is an anomaly [...] <.« (Ebd.: 703)

Meinecke, Tawada und Trojanow versuchen in diesem Sinne in ihren Texten, Perspektiven auf Slums einzunehmen, die diese nicht als homogenes und negativ kodiertes Anderes konstruieren. Diese Transformation des Blicks vollzieht sich vor allem dadurch, dass Slums in diesen Texten als Räume kultureller Praktiken entworfen werden, die progressiv besetzt und in über die Slums hinausgehende Netzwerke eingebunden sind. Was die Autor*innen in Slums finden, unterscheidet sich jedoch nicht grundsätzlich von den Phänomenen, mit denen sie sich auch jenseits von Slums beschäftigen: Ihr Blick verbleibt in einer etischen (im Gegensatz zu einer emischen), den Slums äußerlichen Perspektive. Meineckes, Tawadas und Trojanows literarische Darstellungen von Slums vollziehen Destabilisierungen der Binarität vom Eigenen und Fremden, die ihre Werke allgemein auszeichnen. Solche literarischen Destabilisierungen von Grenzsetzungen ähneln zudem einer alternativen Theoretisierung von Slums, die diese nicht mehr als isolierte Räume fasst, sondern betont, dass Slums durch sich ständig verschiebende Grenzen charakterisiert sind (vgl. Pike 2007: 203-207).

Darstellungen von Slums lassen sich in der deutschsprachigen Literatur vielfältig nachweisen, so zum Beispiel in Egon Erwin Kischs in den 1920er und 1930er Jahren entstandenen Reportagen, die in die Armenviertel Europas, Asiens und Nordamerikas führen (vgl. Schaub 2019a: 214-229), oder in Günter Grass' Zunge zeigen, das während seines Indienaufenthalts in den Jahren 1986 und 1987 entstand (vgl. Grass 1988). Insofern Tawadas Bioskoop der Nacht (2002), Trojanows Die Abschaffung der Armut (2004) und Meineckes Lookalikes (2011) allerdings im Zuge der Neupopularisierung des Begriffs Slum geschrieben wurden und dieser Diskurs in den Texten kritisch aufgegriffen wird, können sie - im Gegensatz zu Kisch und Grass - als Reaktionen auf den neuen Diskurs über Slums seit der Jahrtausendwende verstanden werden. Diese Auseinandersetzung mit Slums erfolgt nun insbesondere vor dem Hintergrund postkolonialer Theoriebildung, die in den hier untersuchten Texten zu einer Neukodierung 
der Kategorie Slum führt. Während Darstellungen von Slums in der Literatur vor allem des 19. und frühen 20. Jahrhunderts in der Forschung diskutiert worden sind (vgl. Poore 2000; Lindner 2004; Kleeberg 2011; Pike 2016), liegt ein Studie zur Gegenwartsliteratur nicht vor. ${ }^{3}$ Meine Analyse der Darstellungen von Slums in Texten von drei vieldiskutierten Gegenwartsautor*innen weist so auf eine Forschungslücke in der literaturwissenschaftlichen Auseinandersetzung mit urbanen Räumen der Globalisierung hin und unterbreitet erste Vorschläge, wie diese geschlossen werden könnte.

\section{TOWNSHIPS IN Kapstadt: Yoko TaWAdas BIOSKOOP DER Nacht}

Slums - genauer die Townships des südafrikanischen Kapstadt - werden zum Gegenstand von Yoko Tawadas Schreiben in Überseezungen (2002), einer Sammlung kurzer Prosatexte, die erstmals die transnationale Topographie ihres Werks über eine zwischen Europa und Ostasien verlaufende »East-West axis« (Yildiz 2007: 78) hinaus ausweitet (vgl. auch Redlich 2017: 199). In Bioskoop der Nacht, dem längsten Text der Sammlung, reist die Ich-Erzählerin nach Südafrika, nachdem sie auf einer Party erfahren hat, dass die Sprache, in der sie träumt, Afrikaans ist - eine Sprache, die sie weder im Wachzustand noch im Traum versteht. Der Text alterniert zwischen Traumsequenzen und in einer Wirklichkeit jenseits des Traums verorteten Passagen, die der ausschließliche Zeitraum sind, in dem Slums vorkommen. Wie in der von Jacques Rancière (vgl. 2003) beschriebenen europäischen Tradition von Reisenarrativen sind auch in der diegetischen Welt von Tawadas Text die Begegnungen mit den Orten der Armen als Begegnungen mit einer authentischeren Wirklichkeit kodiert. Die Konfrontation der Ich-Erzählerin mit den Slums Kapstadts erfolgt dabei in zwei Schritten. Zuerst erblickt sie diese aus dem Taxi heraus, später begibt sie sich in einen Slum, als sie im Rahmen ihres Afrikaanskurses an einer Exkursion teilnimmt. Diese soll, wie ihr Leiter - Herr Tolk - erklärt, »ein anderes Bild vom Township [...] vermitteln als das in der ausländischen Presse, die es bloß als Hölle darstellte.« (Tawada 2002: 77) Tolk will also das medial produzierte Bild der Townships mit einer alternativen, der dortigen Lebenswirklichkeit angemesseneren Vorstellung dieser Orte konfrontieren. Durch ihre positive Bezugnahme auf diese »Absicht« (ebd.) Tolks positioniert die Ich-Erzählerin ihre eigene Sicht der Slums als eine authentischere Darstellung. Diese weist die pathologisierenden Kodierungen von Slums zurück, soll sie Letztere doch nicht »bloß als Hölle darstell[en] «. ${ }^{4}$

Die Ich-Erzählerin entwirft diese alternative Vorstellung, indem sie die Erklärungen Tolks wiedergibt, die Orte beschreibt, die er ihr zeigt, und einem Gespräch mit einer Slumbewohnerin namens Vicky viel Platz einräumt. Slumbe-

3 | Allerdings ist eine neuere filmwissenschaftliche Studie erschienen, die auch die Gegenwart behandelt (vgl. Krstić 2016).

4 | Die Konstruktion eines "andere[n] Bild[es]" der Townships ist Teil einer allgemeineren Verfremdung des touristischen Blicks auf Südafrika (vgl. Maltzan 2012: 191). 
wohnerinnen wie Vicky, »eine der Frauen, die das Besichtigungsprojekt von Herrn Tolk unterstützten « (ebd.), erscheinen somit als Trägerinnen eines Wissens, das dem der ausländischen Besucherin überlegen ist. Sie lenken den Blick der Ich-Erzählerin durch ihre eigenen Perspektiven. Da Tawada diese ihre Darstellung des Townships dominieren lässt, erscheinen Slums als das Normale und Gewohnte und nicht als eine »anomaly« (Gilbert 2007: 703). Jedoch geht es keineswegs darum, die Geschichte der Apartheid oder die Gewalt und Armut unsichtbar zu machen, denen viele schwarze Südafrikaner*innen ausgesetzt sind und die im Ausland synonym für Townships stehen. Diese Themen bleiben in den Schilderungen Tolks zentral; Bioskoop der Nacht kann mithin als Tawadas erste intensive Auseinandersetzung mit dem Verhältnis von Raum, Segregation und der performativen Kraft von race verstanden werden (vgl. Redlich 2017). Entscheidend ist, dass Tawada ihren erzählten Slum nicht auf ein solches Narrativ reduziert. Das von der Ich-Erzählerin besuchte Township wird bei Tawada durch die Perspektiven Tolks und Vickys sowie die Beobachtungen der Ich-Erzählerin zu einem Ort des unspektakulär Alltäglichen. So schildert das erzählende Ich den Bau informeller Häuser: »Neuankömmlinge bauten ihre Wohnräume zuerst aus Pappen, die sie auf der Straße gesammelt hatten. Dann sparten sie Geld, kauften sich nach und nach Holz, Ziegelsteine oder Glasscheiben und ersetzten die Pappen durch die neuen Materialien.« (Tawada 2002: 78) Diese Beschreibung suggeriert weder Verfall noch Stagnation, also Zuschreibungen, die mit der vermeintlich pathologischen Slumkultur verbunden werden, sondern rationale Planung und Entwicklung.

Bioskoop der Nacht verfremdet die hegemoniale Vorstellung von Slums zudem dadurch, dass der Text erstens die Townships durch einen Vergleich in eine Imagination transnationaler Urbanität integriert, sie zweitens als Räume gelebter Mehrsprachigkeit und drittens als Orte kosmopolitischer Alltagspraktiken entwirft. Bereits die erste Begegnung mit den Slums bedeutet für die Ich-Erzählerin eine Konfrontation mit deren vorherrschender medialer Konstruktion. Letztere unterläuft die Ich-Erzählerin jedoch sofort, indem sie bemerkt, dass sie die Townships »irgendwie« an Großstädte Ostasiens »erinner[n]«:

Bald tauchte etwas auf, es sprang in meine Augen: direkt neben der Autobahn viele Schachteln aus Blech und Holz, selbstgebastelte Hütten, hunderte, tausende, bunt, ein Müllpalast, erschreckend, faszinierend. Wie nennt man so etwas? Das Fernsehen hat es uns beigebracht, Slums, Flüchtlingscamps. Irgendwie erinnerte das Zusammenwachsen der Einzelteile auch an ostasiatische Großstädte. Eine Art organische Unordnung. (Ebd.: 72)

Durch diesen Vergleich der aus Japan stammenden und in Deutschland lebenden Ich-Erzählerin wird zum einen eine architektonische Ähnlichkeit zwischen ostasiatischen und südafrikanischen Städten behauptet, wodurch die Townships Südafrikas nicht als das ganz Andere der entwickelten Welt erscheinen, sondern 
als Teil eines globalen Zusammenhangs. ${ }^{5}$ Der Vergleich destabilisiert zum anderen eine dichotome Gegenüberstellung der Welt der Slums und der Welt der IchErzählerin, insofern Letztere eine Dimension ihres eigenen Erfahrungsraums im Erscheinungsbild der Townships wiedererkennt (vgl. Maltzan 2012: 191).

Verfremdet also bereits diese aus der Distanz erfolgende Annäherung an die Slums deren medial produziertes Bild, setzt sich die literarische Neukonstruktion der Slums fort, als sich die Ich-Erzählerin in ein Township begibt. Die Neukodierung erfolgt durch die Charakterisierung der Figur Vicky, die einen mehrsprachigen Alltag führt. Der Slum, für den Vicky im Text beispielhaft steht, wird somit zum multilingualen Interaktionsraum: Vicky

schaltete das Radio an. Eine junge, männliche Stimme strömte heraus, sprach zuerst Englisch, begann dann, ohne eine Schnittstelle bemerken zu lassen, Afrikaans zu sprechen, ging übergangslos wieder zurück, nein, es war kein Englisch mehr, was waren diese Wörter, Afrikaans natürlich, nein, wieder nicht, etwas ganz anderes [...].

Vicky lachte und erklärte mir, es sei die Sprache Xosa, die Sprache des gleichnamigen Stammes. Vicky gehörte auch zum Stamm Xosa. (Tawada 2002: 78f.)

Während diese Mehrsprachigkeit Vorstellungen von Slums als homogenen Räumen destabilisiert und sie als Orte des Zusammenflusses verschiedener Sprachen und Kulturen evoziert, erzeugt Tawada das Township zugleich als Raum kosmopolitischer Alltagspraktiken. Vicky verwendet nämlich Familienmetaphern nicht nur jenseits der biologischen Familie, sondern auch jenseits der rassistischen Unterteilungen, die die Geschichte Südafrikas wesentlich charakterisieren (vgl. Redlich 2017). Als bei Vicky plötzlich »[e]in hellblonder, magerer Mann [...] ins Haus [schleicht], ohne an die Tür zu klopfen«, erklärt Vicky: »Das ist mein kleiner Bruder John. Natürlich ist er nicht wirklich mein Bruder, aber er besucht mich jeden Tag.« (Tawada 2002: 79)

Durch seine Konstruktion der Townships unterminiert Bioskoop der Nacht auf drei verschiedenen Ebenen - Urbanität, Sprache, race - die Binarität vom Eigenen und Fremden. Damit wird der Slum zum beispielhaften Raum eines Themas, mit dem sich Tawada in diesem Text auch jenseits der Townships sowie grundsätzlich in ihrem Werk beschäftigt. In ihrer Lektüre des Textes hat Yasemin Yildiz gezeigt, dass Tawada ihre Ich-Erzählerin als in Afrikaans träumende, in Deutschland lebende und aus Japan stammende Figur so konstruiert, dass der Text die Annahme zurückweist, man könne nur in einer Sprache und Nation beheimatet sein. Die Konstruktion des Textes entlang der Träume in Afrikaans und der Südafrikareise ersetzt die Wahl zwischen Deutsch und Japanisch durch eine dritte Möglichkeit: »Tawada explodes the binary of existing either in the mother tongue or in the foreign language (Yildiz 2007: 78). Dabei verstärkt die Wahl von Afrikaans die Zurückweisung von rassistischen und

5 | Die Einordnung Kapstadts in einen transnationalen Kontext erfolgt auch mit Bezug auf Kapstadts Downtown, wobei nun Los Angeles Vergleichsstadt ist (vgl. Tawada 2002: 73). 
nationalsprachlichen Reinheitsphantasien zugunsten der Annahme einer hybriden Wirklichkeit, insofern diese vom Holländischen abgeleitete und mit rassistischer Herrschaft verbundene Sprache tatsächlich »a hybrid language [ist] which has incorporated lexical and syntactical structures of German, English, Portuguese, Malay, Xhosa, and various other African languages.« (Ebd.: 85) Indem Kapstadts Slums zu einem Raum werden, in dem die Binarität vom Eigenen und Fremden destabilisiert, »die Dreieinigkeitslehre von einer Sprache, einer Nationalität und einem Staat entkräftet« (Brandt/Shyns 2010: 538) und »das Bewusstsein für die Notwendigkeit der Auflösung von Grenzen, Zuordnungen und Vorurteilen [geschärft]« (Maltzan 2012: 193) wird, geht die Neukodierung der Slums mit der literarischen Bestätigung von sprach- und kulturtheoretischen Positionen einher, die für Tawadas Schreiben als wesentlich gelten können. Tawadas neuartiger Blick auf den südafrikanischen Slum findet an diesem Ort insbesondere das, was ebenso in anderswo spielenden Texten zur Darstellung kommt. ${ }^{6}$ Lässt sich also positiv anmerken, dass Tawadas Text einer Tradition der pathologisierenden Stereotypisierung von Slums entgegenarbeitet, kann zugleich problematisierend darauf hingewiesen werden, dass ihr Text im Zuge dieser Neukodierung diese Orte mit etischen, also der Slumkultur selbst äußerlichen, Interpretationsmustern und -kategorien fasst, welche von der Autorin zudem stark (kultur-)politisch aufgeladen werden. Dies ist ein Befund, der auch für die folgenden zwei Beispiele gilt.

\section{Slums in Mumbai: Ilija Trojanows Die Abschaffung der Armut (2004)}

Erstmals 2004 in Wespennest erschienen, gehört Ilija Trojanows Reportage Die Abschaffung der Armut zu seinem umfangreichen Werk kleiner Prosatexte, das im Gegensatz zu den großen Reiseerzählungen des in Bulgarien geborenen, in Deutschland und Kenia aufgewachsenen und weitgereisten Autors bisher eher wenig Beachtung in der Forschung gefunden hat (vgl. Kraenzle 2012: 127). Der Text berichtet auf Basis von Beobachtungen, die laut Untertitel von 1998 bis 2003 über die Slums der indischen Megastadt Mumbai gemacht wurden. Letztere werden durch einen den Text durchziehenden Gegensatz zwischen Reichen und Armen in den Zusammenhang nationaler wie globaler sozioökonomischer Stratifikation gestellt und damit als Teil einer transnationalen Konstellation aufgerufen.

Trojanow wendet sich gegen einen Diskurs über Slums, der diese in Form von »Schreckensbilder[n]« produziert, ihren Bewohner*innen Handlungsfähigkeit abspricht und sie als homogene Räume konstruiert. Ausdrücklich wird Letz-

6 | Auch ihre Darstellung von Berlin betont Multilingualität und Transkulturalität (vgl. Peters 2012: 242-246). 
teres als Effekt der Rhetorik einer neoliberalen Globalisierung markiert, die das Globale als homogen und abstrakt erzeugt: ${ }^{7}$

Slum. So lautet das Wort. S-L-U-M, vier Buchstaben, mit denen das Leben von den meisten Bewohnern Bombays auf einen täuschenden, einen völlig unklaren gemeinsamen Nenner gebracht wird. Slum - mal beschwört das Wort Schreckensbilder von Schmutz, Armut und Krankheit herauf, mal deutet es mit einem empörten und zugleich resignierten Finger auf halbverhungerte, verwahrloste Opfer, die schutzlos und ohnmächtig vor sich hin vegetieren. Durch seine häufige Verwendung hat das Wort wie kaum ein anderes seine eigene virtuelle Wirklichkeit geschaffen. Slum assoziiert eine Einheitlichkeit, die der Diskurs des Globalismus von seinen Kategorien fordert - Slum ist eine statistische Größe. (Trojanow 2010a: 67)

Wenn dieses Bild der Slums hier mit einer »virtuelle[n] Wirklichkeit« verbunden wird, die von der Wirklichkeit des Slums abstrahiert und diesen bloß als »statistische Größe« versteht, dann trifft sich diese »virtuelle Wirklichkeit« in der Reportage damit, wie die Lebenswelten der Reichen gestaltet sind. Symptomatisch sind hierfür hermetisch abgeriegelte golf villages, deren »Landschaft [...] aus verbesserter Natur [besteht]《 (ebd.: 68) und die über einen »Miniaturozean samt artifiziellen Wellen und einem künstlichen Strand« (ebd.: 69) verfügen. Trojanows Reportage hebt diese künstliche Wirklichkeit der Reichen bereits im ersten Absatz hervor. Dort wird die »Scheinwelt« einer »von Kunstrasen umgebenen « Villa auf einem Wolkenkratzer beschrieben, von wo ein »Ausblick « auf das »Wirrwarr der Stadt« aus räumlicher Distanz möglich ist (ebd.: 65).

Diese Wirklichkeitserfahrung der Eliten geht einher mit ihrer Selbstabschottung von der von grenzüberschreitenden Interaktionen geprägten Welt der Armen. Dabei wird diese Selbstabschottung über Mumbai und Indien hinaus globalisiert, indem der Text diese Praxis zum einen mit der freilich in erster Linie rassistisch motivierten »Laager-Mentalität« (ebd.: 66; Hervorh. i.O.) der Buren in Südafrika und zum anderen mit den Lebensverhältnissen der Armen in den USA vergleicht (vgl. ebd.: 74). Selbstabschottung, so die Deutung des Erzählers, führt zu einem Missverstehen der urbanen Wirklichkeit Mumbais, insofern der distanzierte und distanzierende Blick der Reichen auf die Stadt von stereotypen Bildern geprägt bleibt: »In der Vorstellung der Wohlhabenden besteht die Topographie Bombays aus einigen sicheren Inseln inmitten eines bedrohlichen Sumpfes von Chaos und Unordnung « (ebd.: 68).

Trojanows Reportage, die der Begegnung mit der Slumwirklichkeit durch ein Verlassen der »sicheren Inseln« abgewonnen ist, insofern sich der Erzähler in die Slums begibt und dort mit den Bewohner*innen spricht, entwirft eine Beschreibung der Slums, die schon deshalb als vermeintlich authentischere Wirklichkeit erscheint, weil sie nicht Teil der artifiziellen Welt der Reichen und

7 | Mit der Kritik einer Verbindung von Homogenisierung, Abstraktion und Globalismus weist Trojanow Ähnlichkeiten zum von Gayatri Chakravorty Spivak (vgl. 2012) skizzieren Planetarismus auf. 
ihrer epistemischen Perspektive ist. Die Abschaffung der Armut besteht aus einer Aneinanderreihung von Szenen und Beobachtungen, die den anfangs kritisch markierten Blick aus der Ferne auf die Slums mit Beobachtungen aus der Nähe konfrontiert und durch diese überwinden soll. ${ }^{8}$ Der Text alterniert auf diese Weise zwischen einer Kritik stereotypisierender Vorstellungen von Slums und dem Bericht über eine andere Wirklichkeit der Slums von Mumbai, die durch die Begegnungen des Erzählers in diesen Slums entsteht. Dabei dekonstruiert die Reportage die Stereotype des Chaos und der Homogenität, die laut Erzähler von der globalen Elite an die Slums herangetragen werden. Dem angeblichen Chaos stellt die Reportage ein Wissen über die extrem ausbeuterisch strukturierte Wohnungsökonomie der Slums und die Eingebundenheit der Slums in transnationale Produktionsketten gegenüber (vgl. ebd.: 67f. u. 72f.). Zugleich schildert sie Slums ausdrücklich als heterogene Stadträume. So wie die Slumbewohner*innen nicht mit einer Stimme sprechen, sondern zum Beispiel verschiedene Meinungen dazu haben, wie mit der Zerstörung ihrer Nachbarschaft umgegangen werden sollte (vgl. ebd.: ${ }_{55}$ f.), so ist der Slum auch Ort verschiedener Religionen, Berufe und sozialer Gruppen sowie unterschiedlicher architektonischer Stile, die in der Regel auf individueller Improvisation basieren:

Im Slum finden sich Tempel, Moscheen und Kirchen. Gelernte und ungelernte Arbeiter, Akademiker und Analphabeten, Muslims und Hindus, Unberührbare und Angehörige höherer Kasten leben auf engstem Raum neben- und miteinander. Wer es zu etwas Wohlstand bringt, bleibt oft wegen der Gemeinschaft dem Viertel treu. Daher die soliden Bauten, die vielstöckigen Wohnhäuser, die aus dem Meer selbsterrichteter Baracken ragen. Doch auch die eklektisch zusammengezimmerten Hütten unterscheiden sich voneinander. Jede Familie hat mit Einfallsreichtum eine Lösung für die Eigenwilligkeiten ihrer jeweiligen Enge gefunden. (Ebd.: 71)

Über das Motiv der Heterogenität hinaus mobilisiert Trojanows Reportage im Bild des Zusammenlebens verschiedenster Menschen und insbesondere des Nebeneinanders verschiedener Religionen auch die kulturelle Imagination von Bombay als kosmopolitischer Metropole. 9 In Trojanows Texten ist Bombay, das 1996 in Mumbai umbenannt wurde, der Gegenort zu »Bunkern einer vermeintlich homogenen Identität« (Trojanow 2010b: 101) und »bot [...] seit jeher verschiedenen Menschen eine Heimat, ohne irgendeiner Gemeinschaft zu eigen zu sein.« (Ebd.: 100) Es ist eine Stadt, die auch dem Autor selbst eine Art Heimat geboten hat, wie er in Nomade aufvier Kontinenten schreibt: »Bombay/Mumbai hieß mich willkommen, so wie es seit Beginn seiner Geschichte unzähligen Hergelockten und Hingetriebenen - Gujaratis, Parsen, Goanern, Keralesen,

8 | Trojanows Narration der Slums folgt hier dem Muster des "(von außen kommende[n]) teilnehmende[n] Beobachter[s]", das Wagner (2015: 207) für sein Schreiben herausgearbeitet hat.

9 | Zu Bombay/Mumbai und Kosmopolitismus vgl. das vierte Kapitel von Prakash 2010. 
Tamilen, Bengalen, Briten, Arabern, Armeniern, Juden und Chinesen - eine Heimstatt geboten [...] hat.« (Trojanow 2008: 103) Der für Bombay vermeintlich charakteristische Kosmopolitismus wird in der Abschaffung der Armut ausdrücklich mit Slums verbunden und gerade nicht mit den homogenen Räumen der Elite. In Trojanows Reportage werden Slums als kosmopolitische und heterogene Stadträume konstruiert.

Verbindet diese Dimension Die Abschaffung der Armut mit Bioskoop der Nacht, lässt sich wie für Tawadas Text auch für Trojanows Reportage feststellen, dass Slums im Sinne der kulturtheoretischen Annahmen und weltanschaulichen Vorstellungen des Autors konstruiert werden. So entwirft Trojanow im Vorwort zu dem Entfesselten Globus seine eigene Textproduktion und die Welt, mit der sich diese auseinandersetzt, als heterogene: Seine Reportagen seien »so unterschiedlich, wie die Reisen und die Sehnsüchte es waren«, und »sie erzählen alle von einer Welt, deren flimmernd ungewisse Vielfalt mich weiterhin gefangen hält und beglückt.« (Trojanow 2010c: 8) Wie in der zitierten Schilderung des Slums deutlich wird, denkt Trojanow Heterogenität nicht im Sinne von essentialistischer Differenz. Sie entsteht vielmehr dadurch, dass verschiedene Menschen »neben- und miteinander « leben, sich also in Kontakt und Austausch befinden. In seiner programmatischen Ansprache Weltbürgertum heute: Rede zu einer kosmopolitischen Kultur betont Trojanow dann auch gegen einen isolationistischen und »weltfremd[en]« Kulturkonservatismus eine »Dynamik von Verschmelzung und Vermischung, die stets zu kultureller Neuerung führen «. ${ }^{10}$ Aus diesem Weltverständnis, das auf den kulturtheoretischen Annahmen der Hybridisierung und der Produktion von Diversität durch kulturelle Interaktion gründet, folgt für Trojanow die Notwendigkeit einer kosmopolitischen Politik: »[I]ch bin fest davon überzeugt, daß wir als Individuen aber auch als Menschheit keine andere Wahl haben als zu Kosmopoliten zu werden, wenn wir in der Globalität gedeihen und als Gemeinschaft auf diesem ausgelaugten Planeten überleben wollen.« (Trojanow 2012: 32) Die Inszenierung von Mumbais Slums funktioniert in der Abschaffung der Armut als Beispiel dafür, dass der von Trojanow geforderte Kosmopolitismus auf einer vermeintlich richtigen Beschreibung der kulturellen Praktiken einer globalisierten Welt beruht, also mehr sei als eine bloße Wunschvorstellung. ${ }^{11}$

10 | Trojanow 2012: 36. Für Rekonstruktionen dieser kulturtheoretischen Annahme Trojanows vgl. Wagner 2015: 188-193, und Smith-Prei 2017.

11 | Trojanows Kosmopolitismus ist ein kritischer, insofern er Kosmopolitismus ins Verhältnis zu Kolonialismus und sozialen Privilegien setzt (vgl. Kraenzle 2012). Zu Trojanows Selbstverständnis als kosmopolitischem Autor vgl. Wagner 2015: 196-204. 


\section{Favelas in Salvador da Bahia: Thomas MeIneckes LOOKalikes}

Wie Überseezungen in Tawadas Werk markiert Thomas Meineckes sechster Roman Lookalikes (2011) eine geographische Ausweitung der diegetischen Welt Meineckes hin zum Globalen Süden. Während Romane wie Hellblau (2001) in erster Linie Europa und Nordamerika in Beziehung zueinander setzen, verbindet die Geschehnisabfolge von Lookalikes Düsseldorf mit Salvador da Bahia in Brasilien, ${ }^{12}$ wohin der Protagonist Thomas Meinecke auf den Spuren Hubert Fichtes reist. ${ }^{13}$ Dies impliziert auch eine Ausweitung der Geographie des black Atlantic von Nord- nach Südamerika (vgl. Meinecke 2011: 73f.), mit der sich Meinecke im Anschluss an Paul Gilroys Standardwerk The Black Atlantic: Modernity and Double Consciousness (1993) immer wieder auseinandersetzt. Es hat außerdem zur Folge, dass Meineckes Beschäftigung mit den Slums von Chicago in Hellblau (vgl. Schaub 2019b) um eine Auseinandersetzung mit den Favelas Brasiliens ergänzt wird. Während die Begegnung mit Slums in Lookalikes im Unterschied zu den Texten Tawadas und Trojanows nicht ausdrücklich als Begegnung mit einer authentischeren Wirklichkeit inszeniert wird - was nicht überrascht bei einem Autor, der jegliche Wirklichkeit als diskursiv ge- bzw. überformt versteht (vgl. Meinecke 2012: 146f.) -, kommt es dennoch jenseits des Romans selbst zu einer solchen Rahmung in Meineckes Sprechen über den Roman. Mit einer Anleihe an das Vokabular der afroamerikanischen Hip-Hop-Kultur, in der the street oftmals als Bild für eine authentische Wirklichkeitserfahrung figuriert, beschreibt Meinecke seine in den Roman eingegangenen »Erlebnisse« in Brasilien als »wirklich street, unvermittelte Realität « ${ }^{14}$

Meineckes Fiktionalisierung der erfahrenen Wirklichkeit der brasilianischen Slums steht in Lookalikes einem stereotypisierenden Diskurs über Slums entgegen. Diese Gegenüberstellung wird dadurch konstruiert, dass der Protagonist Meinecke im Verlaufe des gesamten Romans immer wieder auf die diskursive Verbindung von Slums mit Gewalt und Unsicherheit verweist, die sowohl den deutschen Blick auf Slums bestimmt (vgl. Meinecke 2011: 97) als auch das Verhalten mancher Brasilianer*innen, zum Beispiel von Taxifahrern, die die Favelas meiden (vgl. ebd.: 128). Der Protagonist hebt diese Assoziation von Slums mit Gefahr zudem als eine Konstante ihrer transnationalen Konstruktion hervor: »Bißchen auch wie in den USA: You don't wanna go there. (Topographie des Terrors.) (Ebd.: 105) Eine Reduktion von Slums auf Orte der Gewalt wird im Ro-

12 | Zur narrativen Struktur von Meineckes Romanen vgl. Picadent 2011: 284.

13 | Laut Meinecke stellt der Brasilienstrang die Fiktionalisierung seiner eigenen Brasilienreise dar, deren "direkt[e] Eindrücke" (Meinecke 2012: 327) die literarische Figur Meinecke notwendig gemacht hätten.

14 | Meinecke 2012: 298. Meinecke erklärt zudem, dass er nur über Orte schreibt, an denen er gewesen ist (vgl. Kreienbrock 2015: 241). Die Wirklichkeit der Diskurse, die sein Schreiben dominiert, wird also durch die Wirklichkeit der Erfahrung des Autors ergänzt. 
man jedoch schon dadurch problematisiert, dass sie der erfahrenen Wirklichkeit der literarischen Figur Meinecke nicht entspricht. Bei seinen Besuchen in Slums wird er nämlich nie bedroht. Insofern er jedoch zumindest aus dem fahrenden Auto heraus einen Polizeieinsatz beobachtet (vgl. ebd.: 152) und darauf hinweist, dass es »ab $21 \mathrm{Uhr}[. .$.$] polizeilich erlaubt [ist], rote Ampeln zu überfah-$ ren, aus Sicherheitsgründen « (ebd.: 123; Hervorh. i.O.), bleibt Gewalt als Teil der Realität der Favelas erhalten.

Die literarische Erzeugung eines Wissens über Slums wird in Lookalikes durch eine narrative Folge plausibilisiert, in der auf den Blick des Protagonisten aus der Ferne direkte Begegnungen mit dem urbanen Raum folgen, welche ein durch Augenzeugenschaft legitimiertes alternatives Wissen ermöglichen. So entwirft der Protagonist die Slums von Salvador da Bahia zuerst aus dem Flugzeug heraus als Teil einer urbanen Topographie, die durch eine am Stadtbild ablesbare Teilung zwischen Armen und Reichen bestimmt ist. Hierbei ordnet Meinecke die Slums durch die zuerst erfolgende Nutzung des Begriffs Slum in eine transnationale Konstellation ein und spezifiziert diese dann durch die Verwendung des Begriffs Favela national: »Das Flugzeug beschreibt eine Schleife über der Baía de Todos os Santos [...]. Erkennbar die Slums, die Favelas, die (wie granuliert) kleinteiligen Quartiere der Armen zwischen den meist schmalen, aber monumentalen Hochhäusern der Reichen.« (Ebd.: 78$)^{15}$

Auf den Blick aus der Ferne folgen mehrere Gänge und Autofahrten des Protagonisten auf den »informellen Wege[n] innerhalb der kaum kartographierten Favelas« (ebd.: 105). Bei diesen wird der Protagonist oftmals von verschiedenen lokalen Führer*innen geleitet (vgl. ebd.: 124). Während diese Gänge durch Meineckes Lektüre von Hubert Fichtes Xango (1976) vorgeformt sind, einem Buch, das sich mit der synkretistischen, aus westafrikanischen und katholischen Elementen bestehenden Religion des Candomblé beschäftigt und für deren Zeremonien in den Favelas der Protagonist Meinecke sich interessiert, so erlaubt dieser thematische Fokus dem Roman, Slums als Räume einer hybriden Kulturproduktion zu kodieren. Diese Hybridität wird zum Beispiel von Wiebke, einer Angestellten des Goethe-Instituts und lokalen Führerin Meineckes, ausdrücklich hervorgehoben:

Einen puren Candomblé könne es nicht geben, denn durch die jahrhundertelange Sklaverei haben sich auf dieser Seite des Atlantiks sämtliche afrikanischen Ethnien verbrüdert [...]. Der Candomblé, den man hier praktiziert, besitzt also afrikanische Wurzeln, ist aber ein brasilianischer Candomblé. Reinheit ist keine Option. (Ebd.: 158; Hervorh. i.0.)

Aufgrund seiner Hybridität wird der Candomblé zum Mittelpunkt der Darstellung der Favelas, denn die literarische Figur Meinecke wie auch der historische Autor »lieb[en] an Kulturen gerade das Hybride« (ebd.: 153). Die spezifische

15 | Meinecke verfolgt die Pluralität von Bezeichnungen für Slums weiter, indem er zeigt, dass Favela kein präferierter Begriff der Slumbewohner*innen ist (vgl. ebd.: 136). 
Konstruktion der Favelas in Lookalikes ist also durch ein allgemeineres Interesse Meineckes an kultureller Hybridität motiviert. ${ }^{6}$ Zugleich erlaubt es die Konzentration auf den Candomblé, die kulturellen Praktiken der Favelas als Teil eines transnationalen Zusammenhangs zu inszenieren. Wie Salvador da Bahia als Stadt (vgl. ebd.: 231f.) sind insbesondere die religiös-musikalischen Praktiken Teil der Kulturen des black Atlantic und eröffnen der literarischen Figur (und dem historischen Autor; vgl. Meinecke 2013: 86 u. 88) Meinecke einen musikalischen Resonanzraum, durch den Nordamerika, Europa, Afrika und Brasilien miteinander verbunden sind:

Auf der Fahrt zurück [von einer Candomblé-Zeremonie; C.S.] ergeht sich Thomas abermals in Vergleichen mit der nordamerikanischen und europäischen Clubkultur. Der Candomblé basiere, wie ein House Club, geradezu architektonisch auf der Musik: Rhythmen dienen als tragende Elemente, als Säulen eines temporären sozialen Raums. (Meinecke 2011: 152)

In den Favelas von Salvador da Bahia und in den von afrodiasporischer Musik geprägten Clubs Nordamerikas und Europas haben die hybriden Musikkulturen des black Atlantic eine ähnliche Bedeutung für die Entstehung temporärer Gemeinschaften. Lookalikes konstruiert die Kultur der Favelas somit nicht als das Andere der Großstädte des Westens, wodurch Meineckes Roman dezidiert die Dichotomie vom Eigenen und Fremden destabilisiert. Im Gegensatz zu ihrer negativen Stereotypisierung sind Slums in diesem Text zudem als kulturell vital und vernetzt kodiert.

\section{Elemente einer Poetologie literarischer Konstruktionen VON SLUMS}

Auf der Grundlage meiner Analysen der Texte Tawadas, Trojanows und Meineckes lassen sich sechs Aspekte herausstellen, in denen sich ihre literarischen Konstruktionen von Slums überschneiden. Diese Elemente sind in den verschiedenen Texten unterschiedlich ausgeprägt. Sie betreffen literarische Darstellungsweisen, thematische Schwerpunkte und weltanschauliche Rahmungen:

1. Die Begegnung mit Slums wird als Begegnung mit der Wirklichkeit kodiert. Durch diese wird eine in den Texten als hegemonial bestimmte, stereotypisierende und pathologisierende sowie oftmals massenmedial erzeugte Konstruktion von Slums zurückgewiesen. Auf diese Weise konstituieren die Texte ein alternatives Wissen über Slums, welches von den Protagonist*innen bezeugt wird - dies gilt umso mehr, als alle drei Texte in Teilen autobiographische Züge tragen. Die Art und Weise, wie dieses alternative Wissen plausibilisiert wird, unterscheidet jedoch die drei Texte voneinander: In

16 | Zu Meineckes Interesse an Hybridität vgl. Schaub 2019b. 
Lookalikes erfolgt die Plausibilisierung über eine Rhetorik der Augenzeugenschaft, die gerade dadurch aufgerufen wird, dass der Protagonist den gleichen Namen trägt wie der Autor des Romans; in Bioskoop der Nacht vollzieht sie sich dadurch, dass die Ich-Erzählerin sich affirmativ auf die Rede der Slumbewohner*innen bezieht und ihnen so einen Wissensvorsprung gegenüber Fremdbeschreibungen der Townships zugesteht; in der Abschaffung der Armut wird das alternative Wissen des Textes dadurch plausibilisiert, dass der hegemoniale Diskurs über Slums als Teil einer »virtuelle[n] Wirklichkeit« inszeniert wird.

2. Alle Texte folgen einem ähnlichen narrativen Muster. Die Auseinandersetzung mit Slums geschieht zuerst durch einen Blick aus der Ferne und in einem zweiten Schritt durch eine Exkursion des/r Protagonist*in in einen Slum. Slums werden somit als Annäherungen von Fremden an diese erzählt. Während diese zwei Schritte in Bioskoop der Nacht und Lookalikes eine narrative Abfolge bilden, oszilliert Trojanows Text jedoch eher kontinuierlich zwischen diesen beiden Perspektiven. Zudem werden die Protagonist*innen in Tawadas und Meineckes Texten von lokalen Führer*innen geleitet, was in der Abschaffung der Armut nicht der Fall ist.

3. Die Protagonist*innen fungieren bei ihren Gängen in die Slums als Grenzüberschreiter*innen. ${ }^{17}$ Sie ähneln in dieser Hinsicht durchaus klassischen Reporterfiguren, die sich zwischen unterschiedlichen Räumen bewegen und in Kontakt mit verschiedenen sozialen Gruppen stehen. ${ }^{18}$ Dabei ist jedoch festzustellen, dass lediglich Trojanow sich der für die Figur des Reporters typischen Form der Reportage bedient.

4. Slums werden nicht als bloß lokale bzw. unverbundene Orte dargestellt. Sie sind vielmehr Räume, in denen sich transnationale und -kulturelle Interaktions- und Austauschprozesse bündeln. Die Texte rufen in diesem Zusammenhang verschiedene Dimensionen der Globalisierung auf: In Trojanows Reportage erscheinen Slums als Räume einer weltweiten sozioökonomischen Stratifikation und eines Kosmopolitismus der Subalternen, in Meineckes Roman werden sie als Teile der Kulturen des black Atlantic verstanden und in Tawadas Text als Knotenpunkte multilingual-kosmopolitischer Alltagspraktiken.

5. Es kommt $\mathrm{zu}$ einer positiven und heterogenisierenden Umkodierung von Slums, durch die diese mit kosmopolitischen Alltagspraktiken, Multilingualität und kultureller Hybridisierung verbunden werden. In einem stärkeren Maße als bei Tawada und Meinecke wird diese Neukodierung bei Trojanow als ein auch politischer Gegensatz zur transnationalen Kultur neoliberaler Eliten positioniert.

17 | Dies betont Meinecke (vgl. 2012: 301) in einem Interview zu Lookalikes.

18 Egon Erwin Kisch, der für Trojanows Reportagepoetik wichtig ist (vgl. Trojanow 2010d: 187f.), formulierte dies früh: „Ein Reporter muß mit allen Kreisen der Gesellschaft Fühlung haben, von der allerhöchsten bis zu den allerniedrigsten." (Kisch 1983: 215) 
6. Die Umkodierung der Slums bedeutet in den drei Texten ihre Erfassung durch die politischen und kulturtheoretischen Vorannahmen, die für die Weltinterpretation der Autor*innen auch jenseits von Slums grundlegend sind. Die Texte legen den Fokus ihrer Konstruktionen von Slums deshalb auf Multilingualität (Tawada), Kosmopolitismus (Trojanow) und Hybridität (Meinecke), obgleich sich diese drei Dimensionen in den Texten freilich nicht säuberlich trennen lassen und in allen zu einem gewissen Maße enthalten sind. Dies bedeutet auch, dass die Slums in diesen Texten mit etischen - im Gegensatz zu emischen - Interpretationsmustern und -kategorien erfasst werden. Dies wäre zwar einerseits zu problematisieren, andererseits allerdings als zumindest in einem geringen Maße unvermeidlich anzuerkennen, da die Texte aus der situierten Perspektive ihrer Autor*innen verfasst sind, welche eben eine den Slums Äußerliche ist.

Auf Grundlage dieser Elemente lassen sich die Texte Meineckes, Tawadas und Trojanows in die von Paul Michael Lützeler beschriebene Tradition einer Reiseliteratur einordnen, in der die Gesellschaften des Globalen Südens »[n]icht mit dem überlegenen, besserwisserischen, ausbeuterischen und missionarischen kolonialen, sondern mit dem offenen, wißbegierigen, solidarischen und gleichwohl kritischen postkolonialen Blick [...] zu erkennen « (Lützeler 1997: 29) versucht werden. Dass selbst dieser postkoloniale Blick noch durch vom beschriebenen Gegenstand relativ unabhängige weltanschauliche und kulturtheoretische Vorannahmen wesentlich geprägt ist, zeigen die politischen und kulturtheoretischen Parameter, mit deren Hilfe Meinecke, Tawada und Trojanow die konkreten kulturellen und sozialen Lebenswelten der Slums konstruieren. Dem postkolonialen Blick dieser Autor*innen ist zudem ein bestimmtes Globalisierungsverständnis eingeschrieben, insofern sie Slums als Teile transnationaler und -kultureller Vernetzungen darstellen. Letztere werden in den Texten nicht durch ein Narrativ vorgestellt, das Globalisierung mit kultureller Homogenisierung verbindet. Die Autor*innen schließen vielmehr an ein - zum Beispiel kulturanthropologisches - Wissen an, das Globalisierungsprozesse als Katalysatoren neuer Formen von Diversität und Hybridität versteht (vgl. Appadurai 1996). Slums erscheinen deshalb in den Texten Meineckes, Tawadas und Trojanows nicht zuletzt als heterogene Stadträume der Globalisierung.

\section{LITERATUR}

Appadurai, Arjun (1996): Modernity at Large. Cultural Dimensions of Globalization. Minneapolis/London.

Brandt, Bettina/Schyns, Désirée (2010): Neu Vernetzt. Yoko Tawadas Bioskoop der Nacht auf Niederländisch. In: Études Germaniques 3, H. 259, S. 535-549.

Brantlinger, Patrick (1985): Victorians and Africans. The Genealogy of the Myth of the Dark Continent. In: Critical Inquiry 12, S. 166-203.

Davis, Mike (2007): Planet of Slums. London/ New York. 
Ette, Ottmar (2016): Writing-between-Worlds. TransArea Studies and the Literatureswithout-a-fixed-Abode. Berlin/Boston.

Gilbert, Alan (2007): The Return of the Slum. Does Language Matter? In: International Journal of Urban and Regional Research 31, H. 4, S. 697-713.

Grass, Günter (1988): Zunge zeigen. Darmstadt.

Kisch, Egon Erwin (1983): Gesammelte Werke in Einzelausgaben. Bd. 9: Mein Leben für die Zeitung. 1926-1947. Journalistische Texte 2. Hg. v. Bodo Uhse u. Gisela Kisch. Fortgeführt v. Fritz Hofmann u. Josef Poláček. Berlin/ Weimar.

Kleeberg, Bernhard (2011): Reisen in den Kontinent der Armut. Ethnographie des Sozialen im 19. Jahrhundert. In: Michael Neumann/Kerstin Stüssel (Hg.): Magie der Geschichten. Weltverkehr, Literatur und Anthropologie in der zweiten Hälfte des 19. Jahrhunderts. Konstanz, S. 29-52.

Kraenzle, Christina (2012): Rewriting Colonial Travelogues. Cosmopolitan Visions and Colonial Legacies in Nomade auf vier Kontinenten/Nomad on Four Continents. In: Julian Preece (Hg.): Ilija Trojanow. Bern, S. 127-152.

Kreienbrock, Jörg (2015): "Ohne Lacan geht natürlich gar nichts - und das ist ganz schön lästig". Ein Interview mit Thomas Meinecke. In: Stefan Höppner/Jörg Kreienbrock (Hg.): Die amerikanischen Götter. Transatlantische Prozesse in der deutschsprachigen Literatur und Popkultur seit 1945. Berlin, S. 233-242.

Krstić, Igor (2016): Slums on Screen. World Cinema and the Planet of Slums. Edinburgh. Lindner, Rolf (2004): Walks on the Wild Side. Eine Geschichte der Stadtforschung. Frankfurt a.M./ New York.

Lützeler, Paul Michael (1997): Einleitung. Der postkoloniale Blick. In: Ders. (Hg.): Der postkoloniale Blick. Deutsche Schriftsteller berichten aus der Dritten Welt. Frankfurt a.M., S. 7-33.

Maltzan, Carlotta von (2012): Magie der Sprache. Yoko Tawada zu Südafrika in Bioskoop der Nacht. In: Dies. (Hg.): Magie und Sprache. Bern, S. 185-195.

Meinecke, Thomas (2011): Lookalikes. Roman. Frankfurt a.M.

Ders. (2012): Ich als Text. Frankfurter Poetikvorlesungen. Frankfurt a.M.

Ders. (2013): Analog. Mit Zeichnungen v. Michaela Melián. Berlin.

Otto, André (2015): Megastadt. Kinematographisches Mumbai und das Rhizom des Abjekten. In: Jörg Dünne/Andreas Mahler (Hg.): Handbuch Literatur und Raum. Berlin/Boston, S. 494-504.

Peters, Laura (2012): Stadttext und Selbstbild. Berliner Autoren der Postmigration nach 1989. Heidelberg.

Picandet, Katharina (2011): Zitatenroman der Gegenwart. Georg Schmid "Roman trouvé", Marcel Beyer "Das Menschenfleisch", Thomas Meinecke "Hellblau«. Frankfurt a.M.

Pike, David L. (2016): Slum. In: Eric Hayot/Rebecca L. Walkowitz (Hg.): A New Vocabulary of Global Modernism. New York, S. 198-213.

Poore, Carole (2000): The Bonds of Labor. German Journeys to the Working World, 1890-1990. Detroit.

Prakash, Gyan (2010): Mumbai Fables. A History of an Enchanted City. Princeton. 
Redlich, Jeremy (2017): Representations of Public Space and the Construction of Race in Yoko Tawada's Bioskoop der Nacht. In: The German Quarterly 90, H. 2, S. 196211.

Rancière, Jacques (2003): Short Voyages to the Land of the People. Aus dem Franz.v. James B. Swenson. Stanford.

Sassen, Saskia (1991): The Global City. New York/London/Tokyo.

Schaub, Christoph (2019a): Proletarische Welten. Internationalistische Weltliteratur in der Weimarer Republik. Berlin/Boston.

Ders. (2019b): The Limits of Connectivity. Literary Knowledge of Globalization in Thomas Meinecke's Hellblau. In: The Germanic Review 94, H. 4, S. 326-344.

Smith-Prei, Carrie (2017): Ilija Trojanow and the Cosmopolitical Public Intellectual. In: Helga Mitterbauer/ Carrie Smith-Prei: Crossing Central Europe: Continuities and Transformations, 1900 and 2000. Toronto/Buffalo/London, S. 251-273.

Spivak, Gayatri Chakravorty (2012): Imperative to Re-imagine the Planet. In: Dies.: An Aesthetic Education in the Era of Globalization. Cambridge/London, S. 335-350.

Tawada, Yoko (2002): Bioskoop der Nacht. In: Dies.: Überseezungen. Tübingen, S. 6191.

Trojanow, Ilija (2008): Nomade auf vier Kontinenten. Auf den Spuren von Sir Richard Francis Burton. München.

Ders. (2010a): Die Abschaffung der Armut. In: Ders.: Der entfesselte Globus. Reportagen. München, S. 65-74.

Ders. (2010b): Bombay Revisited. In: Ders.: Der entfesselte Globus. Reportagen. München, S. 98-109.

Ders. (2010c): Vorwort. In: Ders.: Der entfesselte Globus. Reportagen. München, S. $7 f$.

Ders. (2010d): Die Wahrheit der verwischten Fakten. In: Ders.: Der entfesselte Globus. Reportagen. München, S. 186-191.

Ders. (2012): Weltbürgertum heute: Rede zu einer kosmopolitischen Kultur/What Being a Citizen of the World Means Today. On Cosmopolitan Culture. Aus dem Dt. v. Seiriol Dafydd. In: Julian Preece (Hg.): Ilija Trojanow. Bern, S. 15-43.

Wagner, Sabrina (2015): Aufklärer der Gegenwart. Politische Autorschaft zu Beginn des 21. Jahrhunderts - Julie Zeh, Ilija Trojanow, Uwe Tellkamp. Göttingen.

World Bank/UNCHS (Habitat) (2000): Cities Alliance for Cities without Slums. Action plan for Moving Slum Upgrading to Scale; online unter: https://www.citiesalliance. org/cities-without-slums-action-plan [Stand: 1.4.2020].

Yildiz, Yasemin (2007): Tawada's Multilingual Moves. Toward a Transnational Imaginary. In: Doug Slaymaker (Hg.): Yoko Tawada. Voices from Everywhere. Lanham, S. 77-89. 
